\title{
Risk of migraine in iron deficiency anemia patients with or without iron supplementation usage: a nationwide database analysis
}

\section{Type}

Research paper

\section{Keywords}

gender difference, iron deficiency anemia, migraine, iron supplementation

\begin{abstract}
Introduction

This study evaluated the association between iron deficiency anemia (IDA) and migraine and investigated the effects of iron supplementation, and comorbid conditions, including cardiovascular, respiratory, hepatic, endocrine, metabolic, and other diseases on migraine development.
\end{abstract}

\section{Material and methods}

All study subjects aged $\geq 20$ years with newly diagnosed IDA in the Taiwan National Health Insurance Database during 2000-2012 were enrolled. We matched IDA and non-IDA subjects according to age and sex at a 1:2 ratio. Our primary outcome was diagnosis of migraine, and the patients were monitored until the end of 2013. A Cox proportional hazards regression model was used to evaluate the risk of migraine events in IDA.

\section{Results}

The cumulative incidence of migraine in patients with IDA was 5.82 per 1,000 person-years, which was higher than that in the comparison group (3.99 per 1,000 person-years) during the follow-up period (log-rank test, $p<0.001$ ). After controlling for potentially confounding factors, the risk of migraine was higher in the IDA group compared with the non-IDA group (the adjusted hazard ratio = $1.68,95 \% \mathrm{Cl}=1.51-1.87, \mathrm{p}<0.001)$. Female and $\leq 50$ year-old IDA patients with or without iron supplementation use had an increased incidence of migraine $(p<0.05)$.

\section{Conclusions}

Our study provided additional evidence of an increased risk of migraine in patients with IDA. We also found that females and $\leq 50$ year-old IDA patients with or without iron supplementation use had an increased risk of migraines. 


\section{Risk of migraine in iron deficiency anemia patients with or without iron supplementation usage: a nationwide database analysis}

Herng-Sheng Lee ${ }^{1 \#}$, Shih-Yu Lee ${ }^{\#}$, Wan-Ting Huang ${ }^{3}$, Solomon Chih-Cheng Chen ${ }^{4,5}$, Hsin-Yi Yang ${ }^{3 *}$

${ }^{1}$ Department of Pathology and Laboratory Medicine, Kaohsiung Veterans General Hospital, Kaohsiung 813, Taiwan

${ }^{2}$ Department of Internal Medicine, Ditmansion Medical Foundation Chia-Yi Christian Hospital, Chia-Yi City, Taiwan

${ }^{3}$ Clinical Medicine Research Center, Ditmanson Medical Foundation Chia-Yi

Christian Hospital, Chia-Yi City 600, Taiwan

${ }^{4}$ Department of Pediatrics, School of Medicine, College of Medicine, Taipei Medical University, Taipei 110, Taiwan

${ }^{5}$ Department of Pediatrics, School of Medicine, College of Medicine, Kaohsiung Medical University, Kaohsiung 807, Taiwan

${ }^{\#}$ Herng-Sheng Lee and Shih-Yu Lee are equal contribute as first author.

*Correspondent author Address correspondence and reprint requests to Dr. Hsin-Yi Yang, Clinical Medicine Research Center, Ditmanson Medical Foundation Chia-Yi Christian Hospital, No. 539, Zhongxiao Rd., East District, Chia-Yi City, Taiwan 60002. Tel: +886-5-2765041 ext. 5563, E-mail: cych13018@gmail.com 


\section{Abstract}

Introduction: This study evaluated the association between iron deficiency anemia (IDA) and migraine and investigated the effects of iron supplementation, and comorbid conditions, including cardiovascular, respiratory, hepatic, endocrine, metabolic, and other diseases on migraine development.

Materials and methods: All study subjects aged $\geq 20$ years with newly diagnosed IDA in the Taiwan National Health Insurance Database during 2000-2012 were enrolled. We matched IDA and non-IDA subjects according to age and sex at a 1:2 ratio. Our primary outcome was diagnosis of migraine, and the patients were monitored until the end of 2013. A Cox proportional hazards regression model was used to evaluate the risk of migraine events in IDA.

Results: The cumulative incidence of migraine in patients with IDA was 5.82 per 1,000 person-years, which was higher than that in the comparison group (3.99 per $1,000$ person-years) during the follow-up period (log-rank test, $p<0.001)$. After controlling for potentially confounding factors, the risk of migraine was higher in the IDA group compared with the non-IDA group (the adjusted hazard ratio $=1.68$, $95 \% \mathrm{CI}=1.51-1.87, p<0.001)$. Female and $\leq 50$ year-old IDA patients with or without iron supplementation use had an increased incidence of migraine $(p<0.05)$. 
Conclusions: Our study provided additional evidence of an increased risk of

migraine in patients with IDA. We also found that females and $\leq 50$ year-old IDA

patients with or without iron supplementation use had an increased risk of

migraines.

Key words: iron deficiency anemia, migraine, iron supplementation, gender difference

\section{Running title}

Iron supplementation usage and migraine in IDA patients 


\section{Introduction}

Migraine is a common chronic, intermittent primary headache disorder characterized by recurrent attacks of moderate-to-severe throbbing or pulsatile sensations on one side of the head lasting 4-72 h [1, 2]. Approximately 1.04 billion adults in the world are affected by migraine [3]. The etiology of migraine is multifactorial, involving genetic, hormonal, and environmental factors [4, 5]. Studies have indicated that many comorbidities are associated with migraine, including cardiovascular disorders, psychiatric disorders, neurological diseases, sleep conditions, and inflammatory conditions [6-9]. Moreover, studies have identified comorbidities associated with specific migraine subgroups, such as iron deficiency anemia (IDA) accompanying menstrually related migraine (MRM) [7, $10]$.

Iron deficiency anemia (IDA) is the dominant type of anemia accounting for $>50 \%$ of anemic cases [11]. In the developed world, IDA is prevalent among $2 \%-5 \%$ of adult men and postmenopausal women [12]. IDA can cause various symptoms, such as fatigue, weakness, anxiety, and shortness of breath, and these symptoms may also trigger migraine $[13,14]$. Furthermore, IDA has been linked to migraine headaches and an increased predisposition to pain $[15,16]$. Recent studies have revealed that iron deficiency (with or without anemia) was associated with 
reduced dopamine transporter density and activity, leading to extracellular dopamine and a decline in the number of dopamine receptors in the striatum [17]. Evidence suggests that dopamine is involved in migraine pathogenicity [18].

Till date, no large-scale population-based study has evaluated the increased risk of migraine in patients with IDA. In the present study, we hypothesized that the incidence of IDA is highly correlated with migraine. The objectives of this study were to evaluate the association between iron deficiency anemia (IDA) and migraine and investigate the effects of iron supplementation and comorbid conditions, including cardiovascular disease, respiratory disease, hepatic disease, endocrine and metabolic disorders, and other diseases on migraine development. 


\section{Materials and Methods}

Taiwan's National Health Insurance (NHI) program was launched as a single-payer system on March 1, 1995. As of 2014, the NHI program provides insurance for $99.9 \%$ of Taiwan's population. Data for this study were gathered from the Longitudinal Health Insurance Database 2005 (LHID2005), a subset of the National Health Insurance Research Database (NHIRD). The LHID2005 comprises 1 million people randomly selected from the NHIRD, and no statistically significant differences in age, sex, or health care costs between the LHID2005 sample group and all enrollees were found according to a National Health Research Institutes report [19]. The LHID2005 contains the demographic data of enrollees, including service records and expenditure claims for outpatient, inpatient, and ambulatory care and data associated with contracted pharmacies for reimbursement purposes. The International Classification of Disease, $9^{\text {th }}$ Revision, Clinical Modification (ICD-9-CM) is used to define disease in this database. The data underlying this study are from the NHIRD, which has been transferred to the Health and Welfare Data Science Center (HWDC). Interested researchers can obtain the data through formal application to the HWDC, Department of Statistics, Ministry of Health and

Welfare, Taiwan. This study was reviewed and approved by the Institutional Review Board of the Ditmanson Medical Foundation, Chia-Yi Christian Hospital 
(CYCH-IRB No: 2018078, November 23, 2018), Taiwan. All patient data from the

NHIRD were anonymized; therefore, the board did not require informed consent from the patients for this study.

A retrospective cohort study design and a study cohort comprising patients aged $\geq 20$ years who had received a diagnosis of IDA (ICD-9-CM Code 280) between 2000 and 2012 was conducted. Patients diagnosed as having IDA before 2000 were excluded to increase the likelihood of identifying new cases. The index date was defined as the date of first IDA diagnosis between 2000 and 2012. To ensure accurate diagnoses of IDA, only patients diagnosed with IDA for at least 2 times during the study period were included in this study. The iron supplementary medication (ATC: B03A) data were also collected. Individuals who had missing data and those who were diagnosed without blood tests were excluded. Subjects without IDA were randomly selected from the LHID2005 and designated the non-IDA group. The non-IDA group was frequency matched with the IDA cohort at a 2:1 ratio for age, sex, and the index year that IDA was diagnosed. The study endpoint was development of migraine (ICD-9-CM code 346). Migraine diagnoses were identified according to the International Classification of Headache Disorders, Second Edition criteria (ICHD-II) [20]. The ICHD-II criteria was shown in supplemental Table S1. We included patients in this group only when migraine was diagnosed after the IDA 
index date. For those who did not have migraine, the last day of follow-up was defined as the date of insurance withdrawal or the last day of the study period (December 31, 2013). The mean follow-up duration for the IDA and non-IDA groups were $7.03 \pm 3.82$ and $7.11 \pm 3.81$ years, respectively. The comorbidities consisted of common medical diseases and disorders that may possibly affect IDA or migraine. We considered the following comorbidities as confounding factors in this study: cardiovascular disease, respiratory disease, hepatic disease, endocrine and metabolic disorders, and other diseases. A comorbidity was defined as having the comorbidity before the IDA index date and included hypertension (ICD-9-CM Code 401-405), diabetes mellitus (DM, ICD-9-CM Code 250), dyslipidemia (ICD-9-CM Code 272), coronary artery disease (CAD, ICD-9-CM Code 410-414), stroke (ICD-9-CM Code 430-438), chronic kidney disease (CKD, ICD-9-CM Code 585), cirrhosis (ICD-9-CM: 571.2, 571.5, and 571.6), hyperthyroidism (ICD-9-CM Code 242), hypothyroidism (ICD-9-CM Code 244), and chronic pulmonary disease (COPD, ICD-9-CM Code 490-496). In addition, sociodemographic factors, including age, sex, urbanization level, and economic status were also considered to be confounding factors. There were four urbanization levels (urban, suburban, rural and remote areas), and economic status was classified into three categories: a monthly insurable wage < new Taiwan dollar (NTD) 19,100 (low-income level), 
NTD 19,100-41,999 (intermediate-income level), and NTD $\geq 42,000$ (high-income level). The current exchange rate is approximately US $\$ 0.0316=$ NTD 1.00 .

The chi-square test and Student's $t$ test were used to compare the differences in the demographic and clinical characteristics between the IDA and non-IDA groups. The Kaplan-Meier method was used to estimate the cumulative incidence of migraine between the two groups, and significance was determined using the log-rank test. The incidence rate was calculated as the number of first diagnoses of migraine per 1,000 person-years. We calculated the hazard ratio (HR) and the accompanying 95\% CI by Cox proportional regression models and adjusted the possible covariates that were mentioned above. Subgroup analyses were used to identify any potential differences in the responses to iron supplementation. All statistical analyses were performed using SPSS for Windows version 21.0 (IBM Corporation, Armonk, NY, USA). R software (R Foundation for Statistical Computing, Vienna, Austria) was used to draw the survival curve. A two-tailed p-value of $<0.05$ was considered statistically significant. 


\section{Results}

\section{Baseline characteristics of the study population}

A total of 26,217 patients diagnosed as having IDA and 52,434 sex- and age-matched controls were included (Table 1). Figure 1 depicts the flowchart for selecting the study population.

Incidence rates and HR analysis of migraine in the patients with IDA

The cumulative incidence of migraine was higher in the patients with IDA than in the comparison group (5.82 per 1,000 person-years vs. 3.99 per 1,000 person-years, respectively; log-rank test, $p<0.001)$ during the follow-up period (Figure 2). After adjusting for age, sex, area, income level, iron supplementation, and comorbidities, the risk of migraine was significantly higher in the IDA group than in the comparison group [adjusted $\mathrm{HR}(\mathrm{aHR})=1.68,95 \% \mathrm{CI}=1.51-1.87$, $p<0.001]$. Multivariate Cox proportional hazards analysis showed that female, rural area, dyslipidemia, and COPD were at a heightened risk of migraine relative to non-IDA group $(p<0.05)$. Moreover, age $>50$ years, hypertension, DM, CKD, and iron supplementation were at a lower risk of migraine $(p<0.05$, Table 2$)$.

Adjusted HRs measured using the multivariate Cox proportional model for patients with migraine associated with IDA and iron supplementation and stratified by sex 
Table 3 presents the sex stratification analysis of the risk of IDA-associated

migraine. In the comparison group, females without iron supplementation use had a higher risk of migraine than the risk of those with iron supplementation use $(\mathrm{aHR}=$ $1.45,95 \% \mathrm{CI}=1.12-1.87)$. Furthermore, females who had IDA with or without iron supplementation use had a higher migraine risk than non-IDA females $(p<0.001)$.

\section{Adjusted HRs measured using the multivariate Cox proportional model}

\section{for patients with migraine associated with IDA and iron supplementation and}

\section{stratified by age}

Non-IDA controls $\leqq 50$ years old without iron supplementation use had a higher risk of migraine than that of non-IDA controls $\leqq 50$ years old with iron supplementation use $(\mathrm{aHR}=1.63$ with $95 \% \mathrm{CI}=1.20-2.22)$. In addition, IDA patients $\leqq 50$ years old with or without iron supplementation use had a higher risk of migraine than that of the non-IDA controls $<50$ years old without iron supplementation use $(p<0.001$, Table 4). 


\section{Discussion}

This nationwide population-based cohort study demonstrated that the risk of migraine was markedly higher for patients with IDA than for the non-IDA group, with an aHR of 1.68. Furthermore, the IDA patients with or without iron supplementation were associated with significantly higher risks of migraine, especially in females or patients $\leq 50$ years old. These results support those of previous reports showing that IDA may be a risk factor for developing migraine. Pamuk et al. stated that IDA patients experienced migraines more frequently, which can be indicative of an underlying relationship between IDA and diseases and central nervous system problems [15]. A similar study was conducted in Iran by Gholamreza-Mirzaee et al. They found that iron supplements might be an effective treatment or prophylaxis in patients with migraine associated with IDA [21]. We also found that migraine was more prevalent in females with IDA than in males with IDA. Previous research has also found that IDA was more common in patients with menstrual migraine and that migraine attacks could be triggered by IDA [10].

In the present study, we revealed that IDA had negative effects on the risk for migraine in patients aged $<50$ years. Former studies have stated that migraine is most commonly diagnosed in individuals aged between 25 and 55 years and its peak is between 30-39 years of age [22]. A previous study suggested that migraines are a 
genetic disorder that runs in the family, which usually occur during childhood near the age of adolescence with a few attacks per year [4]. Then, it progresses into a few attacks per week between the ages of 22 and 55 years, after which the symptoms of migraine may be less intense, milder, or may disappear altogether [5]. Moreover, a 12 year retrospective follow-up study that included 374 patients with migraine from Sweden found that $29 \%$ of the patients no longer had the disorder as they aged, and the remaining $71 \%$ had fewer attacks that were less frequent and caused milder pain [23]. Previous study was also indicated that female after menopause, the prevalence of migraine falls by approximately one-half, at least partly as a result of hormonal stability $[24,25]$. This decrease in migraines with increasing age may be because of altered brain structure and functionality caused by repeated headache episodes [26, 27]. Additionally, during the aging process, the brain begins to lose cells and shrinks and may exhibit a decrease in several neurological functions and signaling pathways [28].

Besides age, our results indicated that females had a higher risk of migraine than males, consistent with previously published results [29, 30]. Significant differences between genders have been found in multiple epidemiological studies of migraine [31]. Migraine is reported to be three times more prevalent in females than in males [32]. A distinction in hormonal balances is the major reason why females 
suffer migraines more frequently than males. Estrogen and progesterone can affect the pain-processing networks and the endothelium involved in the pathophysiology of migraine $[33,34]$. More recent studies have proved that the drop in estrogen may lead to the vasodilatation of cranial vasculature and a sensitization of trigeminal nerves, which can trigger migraine $[35,36]$. A study also found that women with migraine experience a faster drop in estrogen levels just before the onset of their period than non-migraineurs [34]. In addition, the estrogen also influence serotonin neurotransmission system and calcitonin gene-related peptide (CGRP) levels making women more predisposed to migraine attacks. Previous research was showed that the level of CGRP were higher in women of reproductive age than in men [37]. Hormonal fluctuations during the female reproductive life cycle (eg., menstruation, pregnancy, and menopause) are known to impact the frequency and intensity of migraine [38]. In addition to hormonal triggers, there are a number of factors contributing to migraine, such as genetic and epigenetic factors, environmental stressors, psychosocial factors, and coping strategies in response to stress and pain $[4,5]$. Psychosocial factors (stress, anxiety, and irritability) have a greater effect on females than on males [39]. These parameters may explain, in part, the differences in prevalence and presentation of migraine between females and males. 
Although there are many causes of migraine, one of these may be iron

deficiency. Several studies have shown an association between iron deficiency and migraine headache in adults $[15,21,40]$. Keivani et al. showed that iron deficiency was more frequent in 15 to 45 year-old women with migraines than in women without migraines [40]. A study in Turkey reported that $36.2 \%$ of iron-deficient patients experienced migraines [15]. Another study in Iran indicated that ferrous sulfate therapy was effective in reducing the monthly frequency and severity of migraine headaches in 15 to 45 year-old women with and without iron deficiency [21]. Previous studies have also indicated that there might be an association between body iron storage status and the incidence of migraine $[15,41]$. Similar to previous findings, our results found that iron supplementation was beneficial for reducing migraine risk in IDA patients. A possible explanation may be that iron deficiency can decrease hemoglobin levels and the blood then is unable to deliver enough oxygen to the brain, which causes migraines [15]. Therefore, it could be important for adults with migraine to have their iron status and serum iron and ferritin levels evaluated.

Studies have also indicated that migraine is associated with comorbidities, including cardiovascular disorders, stroke, psychiatric disorders, sleep conditions, inflammatory conditions, and chronic pain [7, 9]. In this research, we found that 
patients with dyslipidemia were at a high risk of migraine. Recent studies have suggested that the intensity and frequency of migraine attacks may be related to the level of blood lipids $[42,43]$. This may be attributed to the fact that dyslipidemia induces platelet aggregation and triggers neurogenic inflammation by inducing changes in serum serotonin and platelet serotonin levels, initiating the cascades of prostaglandins (mainly PGE2) and leukotrienes (LT) formation [44]. These changes lead to vasodilatation and migraine headache. Our research also found that patients with COPD were at a high risk of migraine. In a cross-sectional study, data from the 2013-2015 National Health Interview Survey $(n=104,926$ adults) indicated associations between migraine and COPD [45]. Another study using multivariate logistic regression analyses suggested that COPD was positively associated with migraine. Patients with COPD have damaged lungs caused by chronic bronchitis and emphysema that hampers the normal functioning of the lungs [46]. These problems along with restricted airflow may cause migraine.

This study had a number of strengths. First, the data were retrieved from the NHIRD, which is highly representative of the general Taiwanese population. Moreover, a large population-based sample size can provide adequate statistical power to show the significance of any associations between factors and an effect and avoids selection bias. However, this study had several limitations that should be 
addressed. First, the incidence rate of migraine may have been underestimated because only patients who sought medical help were enrolled in the study. Second, each patient's clinical information, such as IDA severity, migraine frequency, and the presence or absence of auras, neuroimaging, or other laboratory results, was not available in the NHIRD. Therefore, it was difficult to evaluate the effect of IDA severity on migraines in this study. Finally, detailed information on lifestyle, individual behavior, psychological status, nutrition status, and environmental effects were not recorded in the NHIRD, all of which might have been confounding factors. Hence, more prospective studies are warranted to further investigate the association between IDA and migraine.

\section{Conclusion}

Our study showed that IDA increased the likelihood of experiencing migraines in the subjects. Moreover, female and $\leq 50$ year-old IDA patients with or without iron supplementation use were found to have an increased risk of migraines. According to our results, iron supplementation was beneficial for reducing the risk of migraine in patients with IDA. It indicates that iron supplements might be an effective treatment or prophylaxis to reduce the risk of migraine in patients with IDA. Clinicians should be aware of the high migraine risk in patients with new IDA 
diagnosis and implement appropriate monitoring of high-risk groups. Future studies are needed to clarify the pathogenesis of IDA on the development of migraine. 


\section{Acknowledgements}

This study was based in part on data from the National Health Insurance

Research Database provided by the Bureau of National Health Insurance and the

Department of Health and Welfare and managed by the National Health Research

Institutes. The interpretation and conclusions contained within do not represent those

of the Bureau of National Health Insurance, Department of Health or National

Health Research Institutes.

\section{Conflict of interest}

The authors declare no conflict of interest. 


\section{References}

1. Ahmed F. Headache disorders: differentiating and managing the common subtypes. Br J Pain 2012; 6: 124-32.

2. Zhang Y, Kong Q, Chen J, Li L, Wang D, Zhou J. International Classification of Headache Disorders 3rd edition beta-based field testing of vestibular migraine in China: Demographic, clinical characteristics, audiometric findings and diagnosis statues. Cephalalgia 2016; 36: 240-8.

3. Mattiuzzi C, Lippi G. Updates on migraine epidemiology. Eur J Neurol 2020; 27: e13.

4. Sutherland HG, Albury CL, Griffiths LR. Advances in genetics of migraine. J Headache Pain 2019; 20: 72.

5. Burstein R, Noseda R, Borsook D. Migraine: multiple processes, complex pathophysiology. J Neurosci 2015; 35: 6619-29.

6. Minen MT, Begasse De Dhaem O, Kroon Van Diest A,et al. Migraine and its psychiatric comorbidities. J Neurol Neurosurg Psychiatry 2016; 87: 741-9.

7. Buse DC, Reed ML, Fanning KM, et al. Comorbid and co-occurring conditions in migraine and associated risk of increasing headache pain intensity and headache frequency: results of the migraine in America symptoms and treatment (MAST) study. J Headache Pain 2020; 21: 23. 
8. Burch RC, Buse DC, Lipton RB. Migraine: Epidemiology, Burden, and Comorbidity. Neurologic clinics 2019; 37: 631-49.

9. Martin VT, Fanning KM, Serrano D, Buse DC, Reed ML, Lipton RB. Asthma is a risk factor for new onset chronic migraine: Results from the American migraine prevalence and prevention study. Headache 2016; 56: 118-31.

10. Vukovic-Cvetkovic V, Plavec D, Lovrencic-Huzjan A, Galinovic I, Seric V, Demarin V. Is iron deficiency anemia related to menstrual migraine? Post hoc analysis of an observational study evaluating clinical characteristics of patients with menstrual migraine. Acta clinica Croatica 2010; 49: 389-94.

11. Grosbois B, Decaux O, Cador B, Cazalets C, Jego P. [Human iron deficiency]. Bull Acad Natl Med 2005; 189: 1649-63.

12. Johnson-Wimbley TD, Graham DY. Diagnosis and management of iron deficiency anemia in the 21st century. Therap Adv Gastroenterol 2011; 4: 177-84.

13. Jarrah SS, Halabi JO, Bond AE, Abegglen J. Iron deficiency anemia (IDA) perceptions and dietary iron intake among young women and pregnant women in Jordan. J Transcult Nurs 2007; 18: 19-27.

14. Ierusalimschy R, Moreira PF. Precipitating factors of migraine attacks in 
patients with migraine without aura. Arq Neuropsiquiatr 2002; 60: 609-13.

15. Pamuk GE, Top MS, Uyanik MS, et al. Is iron-deficiency anemia associated with migraine? Is there a role for anxiety and depression? Wien Klin Wochenschr 2016; 128: 576-80.

16. Gur-Ozmen S, Karahan-Ozcan R. Iron Deficiency Anemia Is Associated with Menstrual Migraine: A Case-Control Study. Pain Med 2016; 17: 596-605.

17. Lozoff B. Early iron deficiency has brain and behavior effects consistent with dopaminergic dysfunction. J Nutr 2011; 141: 740s-6s.

18. Barbanti P, Fofi L, Aurilia C, Egeo G. Dopaminergic symptoms in migraine. Neurol Sci 2013; 34 Suppl 1:S67-70.

19. National Health Insurance Research Database. (Accessed October 22, 2019.). Available at: http://nhird.nhri.org.tw/en/Data_Subsets.html.

20. Headache Classification Subcommittee of the International Headache S. The International Classification of Headache Disorders: 2nd edition. Cephalalgia 2004; 24: 9-160.

21. Gholamreza-Mirzaee M, Kheiri S, Khosravi S, Khoshdel A, Keyvani Z, Amini Z. Iron therapy and migraine headache. J Shahrekord Univ Med Sci $2012 ; 13$.

22. Bigal ME, Liberman JN, Lipton RB. Age-dependent prevalence and clinical 
features of migraine. Neurology 2006; 67: 246-51.

23. Dahlof CG, Johansson M, Casserstedt S, Motallebzadeh T. The course of frequent episodic migraine in a large headache clinic population: a 12-year retrospective follow-up study. Headache 2009; 49: 1144-52.

24. Martin VT, Pavlovic J, Fanning KM, Buse DC, Reed ML, Lipton RB. Perimenopause and menopause are associated with high frequency headache in women with migraine: results of the American migraine prevalence and prevention study. Headache 2016; 56: 292-305.

25. Wang SJ, Fuh JL, Lu SR, Juang KD, Wang PH. Migraine prevalence during menopausal transition. Headache 2003; 43: 470-478.

26. Bravo TP. Headaches of the elderly. Curr Neurol Neurosci Rep 2015; 15: 30.

27. Straube A, Andreou A. Primary headaches during lifespan. J Headache Pain $2019 ; 20: 35$.

28. Mattson MP, Arumugam TV. Hallmarks of Brain Aging: Adaptive and Pathological Modification by Metabolic States. Cell Metab 2018, 27: 1176-99.

29. Allais G, Chiarle G, Sinigaglia S, Benedetto C. Menstrual migraine: a review of current and developing pharmacotherapies for women. Expert Opin Pharmacother 2018; 19: 123-36. 
30. Risal A, Manandhar K, Holen A, Steiner TJ, Linde M. Comorbidities of psychiatric and headache disorders in Nepal: implications from a nationwide population-based study. J Headache Pain 2016; 17: 45.

31. Buse DC, Loder EW, Gorman JA, et al. Sex differences in the prevalence, symptoms, and associated features of migraine, probable migraine and other severe headache: results of the American Migraine Prevalence and Prevention (AMPP) Study. Headache 2013; 53: 1278-99.

32. GBD 2016 Disease and Injury Incidence and Prevalence Collaborators. Global, regional, and national incidence, prevalence, and years lived with disability for 328 diseases and injuries for 195 countries, 1990-2016: a systematic analysis for the Global Burden of Disease Study 2016. Lancet 2017; 390: 1211-59.

33. Brandes JL. The influence of estrogen on migraine: a systematic review. JAMA 2006; 295: 1824-30.

34. Pavlovic JM, Allshouse AA, Santoro NF, et al. Sex hormones in women with and without migraine: Evidence of migraine-specific hormone profiles. Neurology 2016; 87: 49-56.

35. Calhoun AH, Batur P. Combined hormonal contraceptives and migraine: An update on the evidence. Cleve Clin J Med 2017; 84: 631-8. 
36. Calhoun A. Combined hormonal contraceptives: is it time to reassess their role in migraine? Headache 2012; 2: 648-60.

37. Aggarwal M, Puri V, Puri S. Serotonin and CGRP in migraine. Ann Neurosci 2012; 19: 88-94.

38. Faubion SS, Batur P, Calhoun AH. Migraine Throughout the Female Reproductive Life Cycle. Mayo Clin Proc 2018; 93: 639-45.

39. Lebedeva ER, Kobzeva NR, Gilev DV, Kislyak NV, Olesen J. Psychosocial factors associated with migraine and tension-type headache in medical students. Cephalalgia 2017; 37: 1264-71.

40. Keivani Z, Mirzaei M, Mahmoudzadeh M, Etemadifar S, Rafieian M. Evaluation the relationship between iron deficiency anemia and migraine headache in patients who referred to neurology clinic of Shahrekord University of Medical Science. Iran J Nurs Res 2010; 23: 37-43.

41. Rainero I, Roveta F, Vacca A, Noviello C, Rubino E. Migraine pathways and the identification of novel therapeutic targets. Expert Opin Ther Targets 2020; 24: $245-53$.

42. Ozcan RK, Ozmen SG. The Association Between Migraine, Metabolic Syndrome, Insulin Resistance, and Obesity in Women: A Case-Control Study. Sisli Etfal Hastan Tip Bul 2019; 53: 395-402. 
43. Tana C, Santilli F, Martelletti P, et al. Correlation between Migraine Severity and Cholesterol Levels. Pain Pract 2015; 15: 662-70.

44. Ghosh KC, Bhattacharya R, Ghosh S, et al. Study to Evaluate the Role of Patent Foramen Ovale in Migraine with Aura and Identification of the Predictors of Aura among Patients Attending the Neurology Outpatient Department at a Tertiary Care Centre of Eastern India. EC Neurology 2018; 10: 285-92.

45. Minen MT, Weissman J, Tietjen GE. The Relationship Between Migraine or Severe Headache and Chronic Health Conditions: A Cross-Sectional Study from the National Health Interview Survey 2013-2015. Pain Med 2019; 20: 2263-71.

46. Wang X, Xing Y, Sun J, et al. Prevalence, Associated Factors, and Impact on Quality of Life of Migraine in a Community in Northeast China. J Oral Facial Pain Headache 2016; 30: 139-49. 


\section{Figure legends}

Figure 1. Flow diagram of the present study from the NHIRD.

Figure 2. Cumulative incidence of migraine in the IDA and non-IDA groups,

compared with a log-rank test. A statistically significant difference exists between the IDA and non-IDA groups $(p<0.001)$ 
Table 1. Demographic characteristics and comorbidities in groups with and without IDA

\begin{tabular}{|c|c|c|c|}
\hline & $\begin{array}{l}\text { IDA Group } \\
\mathrm{N}=26,217\end{array}$ & $\begin{array}{c}\text { Non-IDA Group } \\
\mathrm{N}=52,434\end{array}$ & $p$-value \\
\hline Age, mean $\pm \mathrm{SD}$ & $51.76 \pm 18.40$ & $51.76 \pm 18.40$ & 0.998 \\
\hline$\leq 50$ & $14698(56.06)$ & $29424(56.12)$ & 0.887 \\
\hline$>50$ & 11519 (43.94) & $23010(43.88)$ & \\
\hline Sex & & & 1.000 \\
\hline Male & $6644(25.34)$ & $13288(25.34)$ & \\
\hline Female & $19573(74.66)$ & $39146(74.66)$ & \\
\hline Area & & & $<0.001$ \\
\hline Urban & $15201(57.98)$ & $32028(61.08)$ & \\
\hline Suburban & $8167(31.15)$ & $15491(29.54)$ & \\
\hline Rural & $1833(6.99)$ & $3086(5.89)$ & \\
\hline Remote area & $1016(3.88)$ & $1829(3.49)$ & \\
\hline Income level & & & $<0.001$ \\
\hline Low & $13332(50.85)$ & $27296(52.06)$ & \\
\hline Intermediate & $11176(42.63)$ & 21435 (40.88) & \\
\hline High & 1709 (6.52) & $3703(7.06)$ & \\
\hline Hypertension & $8350(31.85)$ & $13438(25.63)$ & $<0.001$ \\
\hline DM & $4532(17.29)$ & $6200(11.82)$ & $<0.001$ \\
\hline Dyslipidemia & 4805 (18.33) & 7849 (14.97) & $<0.001$ \\
\hline CAD & $4538(17.31)$ & 6785 (12.94) & $<0.001$ \\
\hline Stroke & 3363 (12.83) & $4732(9.02)$ & $<0.001$ \\
\hline CKD & $1501(5.73)$ & $844(1.61)$ & $<0.001$ \\
\hline Cirrhosis & $999(3.81)$ & $425(0.81)$ & $<0.001$ \\
\hline Hyperthyroidism & $865(3.30)$ & $1177(2.24)$ & $<0.001$ \\
\hline Hypothyroidism & $336(1.28)$ & $423(0.81)$ & $<0.001$ \\
\hline COPD & 6185 (23.59) & $9698(18.50)$ & $<0.001$ \\
\hline Iron supplementation & $16802(64.09)$ & $2504(4.78)$ & $<0.001$ \\
\hline
\end{tabular}

Data are presented as the mean $\pm \mathrm{SD}$ or number (percentage, \%); $\mathrm{DM}=$ diabetes mellitus; $\mathrm{CAD}=$ coronary artery disease $\mathrm{CKD}=$ chronic kidney disease COPD = chronic obstructive pulmonary disease. 
Table 2. Cox model-measured hazard ratios and $95 \%$ confidence interval of migraine associated with IDA and covariates

\begin{tabular}{|c|c|c|c|c|c|c|c|}
\hline \multirow[t]{2}{*}{ Variables } & \multicolumn{3}{|c|}{ IDA } & \multicolumn{3}{|c|}{ Non-IDA } & \multirow{2}{*}{$\begin{array}{c}\text { Adjusted HR } \\
(95 \% \mathrm{CI})\end{array}$} \\
\hline & Event & PY & Rate $^{\#}$ & Event & PY & Rate $^{\#}$ & \\
\hline Migraine & 1073 & 184373.67 & 5.82 & 1490 & 373241.22 & 3.99 & $1.68(1.51-1.87) * * *$ \\
\hline Age $-\leq 50$ & 831 & 107922.02 & 7.70 & 1087 & 219576.05 & 4.95 & 1.00 (ref.) \\
\hline$->50$ & 242 & 76451.65 & 3.17 & 403 & 153665.17 & 2.62 & $0.59(0.52-0.66) * * *$ \\
\hline Sex - Male & 98 & 44033.65 & 2.23 & 161 & 88592.38 & 1.82 & 1.00 (ref.) \\
\hline - Female & 975 & 140340.03 & 6.95 & 1329 & 284648.84 & 4.67 & $2.30(2.01-2.63) * * *$ \\
\hline Area - Urban & 631 & 107172.02 & 5.89 & 917 & 227405.87 & 4.03 & 1.00 (ref.) \\
\hline - Suburban & 331 & 57291.88 & 5.78 & 421 & 110426.96 & 3.81 & $1.03(0.95-1.13)$ \\
\hline - Rural & 73 & 12805.61 & 5.70 & 94 & 22263.52 & 4.22 & $1.25(1.06-1.47)^{* *}$ \\
\hline - Remote area & 38 & 7104.16 & 5.35 & 58 & 13144.87 & 4.41 & $1.17(0.95-1.44)$ \\
\hline Income level - Low & 504 & 93723.21 & 5.38 & 740 & 196017.5 & 3.78 & 1.00 (ref.) \\
\hline - Intermediate & 492 & 78598.78 & 6.26 & 626 & 151375.19 & 4.14 & $1.01(0.93-1.10)$ \\
\hline - High & 77 & 12051.68 & 6.39 & 124 & 25848.53 & 4.80 & $1.07(0.92-1.24)$ \\
\hline Hypertension - No & 898 & 131754.42 & 6.82 & 1255 & 289118.45 & 4.34 & 1.00 (ref.) \\
\hline - Yes & 175 & 52619.25 & 3.33 & 235 & 84122.77 & 2.79 & $0.84(0.73-0.97)^{*}$ \\
\hline DM - No & 986 & 156457.96 & 6.30 & 1396 & 335417.79 & 4.16 & 1.00 (ref.) \\
\hline - Yes & 87 & 27915.72 & 3.12 & 94 & 37823.43 & 2.49 & $0.75(0.63-0.88)^{* *}$ \\
\hline Dyslipidemia - No & 954 & 156655.30 & 6.09 & 1331 & 329107.70 & 4.04 & 1.00 (ref.) \\
\hline - Yes & 119 & 27718.37 & 4.29 & 159 & 44133.52 & 3.60 & $1.26(1.10-1.46)^{* *}$ \\
\hline CAD - No & 966 & 155899.47 & 6.20 & 1359 & 331987.76 & 4.09 & 1.00 (ref.) \\
\hline - Yes & 107 & 28474.21 & 3.76 & 131 & 41253.46 & 3.18 & $1.14(0.97-1.33)$ \\
\hline Stroke - No & 1014 & 164226.33 & 6.17 & 1421 & 345445.03 & 4.11 & 1.00 (ref.) \\
\hline - Yes & 59 & 20147.35 & 2.93 & 69 & 27796.19 & 2.48 & $0.84(0.69-1.03)$ \\
\hline CKD - No & 1051 & 175040.47 & 6.00 & 1482 & 368683.62 & 4.02 & 1.00 (ref.) \\
\hline - Yes & 22 & 9333.20 & 2.36 & 8 & 4557.59 & 1.76 & $0.64(0.45-0.93)^{*}$ \\
\hline Cirrhosis - No & 1058 & 178576.68 & 5.92 & 1482 & 370745.68 & 4.00 & 1.00 (ref.) \\
\hline - Yes & 15 & 5796.99 & 2.59 & 8 & 2495.53 & 3.21 & $0.84(0.55-1.27)$ \\
\hline Hyperthyroidism - No & 1029 & 178921.00 & 5.75 & 1463 & 366467.96 & 3.99 & 1.00 (ref.) \\
\hline - Yes & 44 & 5452.67 & 8.07 & 27 & 6773.26 & 3.99 & $0.99(0.78-1.27)$ \\
\hline Hypothyroidism - No & 1052 & 182464.64 & 5.77 & 1482 & 371023.02 & 3.99 & 1.00 (ref.) \\
\hline - Yes & 21 & 1909.04 & 11.00 & 8 & 2218.20 & 3.61 & $1.33(0.92-1.93)$ \\
\hline COPD - No & 870 & 146049.19 & 5.96 & 1255 & 315021.44 & 3.98 & 1.00 (ref.) \\
\hline - Yes & 203 & 38324.49 & 5.30 & 235 & 58219.78 & 4.04 & $1.33(1.19-1.49) * * *$ \\
\hline \multicolumn{8}{|l|}{ Iron supplementation } \\
\hline - No & 343 & 59456.23 & 5.77 & 1424 & 351321.93 & 4.05 & 1.00 (ref.) \\
\hline - Yes & 730 & 124917.45 & 5.84 & 66 & 21919.29 & 3.01 & $0.81(0.72-0.91)^{* * *}$ \\
\hline
\end{tabular}

$\mathrm{DM}=$ diabetes mellitus; $\mathrm{CAD}=$ coronary artery disease; $\mathrm{PY}=$ person-years; Rate $^{\#}=$ incidence rate in per 1,000 person-years; $\mathrm{CKD}=$ chronic kidney disease; $\mathrm{COPD}=$ chronic obstructive pulmonary disease. 
Table 3. Adjusted HRs measured using the multivariate Cox proportional model for patients with migraine associated with IDA and iron supplementation and stratified by sex

\begin{tabular}{|c|c|c|c|c|c|c|}
\hline \multicolumn{7}{|l|}{ Male } \\
\hline IDA & $\begin{array}{c}\text { Iron } \\
\text { supplementation }\end{array}$ & Event & PY & Rate $^{\#}$ & IRR (95\% CI) & Adjusted HR $(95 \% \mathrm{CI}) \ddagger$ \\
\hline- & + & 6 & 3564.42 & 1.68 & 1.00 (ref.) & 1.00 (ref.) \\
\hline- & - & 155 & 85027.95 & 1.82 & $1.08(0.48-2.45)$ & $0.86(0.38-1.95)$ \\
\hline+ & + & 44 & 22739.86 & 1.93 & $1.15(0.49-2.70)$ & $0.97(0.41-2.29)$ \\
\hline+ & - & 54 & 21293.79 & 2.54 & $1.51(0.65-3.50)$ & $1.17(0.50-2.74)$ \\
\hline \multicolumn{7}{|l|}{ Female } \\
\hline IDA & $\begin{array}{c}\text { Iron } \\
\text { supplementation }\end{array}$ & Event & PY & Rate $^{\#}$ & $\operatorname{IRR}(95 \% \mathrm{CI})$ & Adjusted HR (95\% CI) \\
\hline- & + & 60 & 18354.87 & 3.27 & 1.00 (ref.) & 1.00 (ref.) \\
\hline- & - & 1269 & 266293.98 & 4.77 & $1.46(1.13-1.89)^{* * *}$ & $1.45(1.12-1.87)^{*}$ \\
\hline+ & + & 686 & 102177.59 & 6.71 & $2.05(1.58-2.67)^{* * *}$ & $2.00(1.54-2.61)^{* * *}$ \\
\hline+ & - & 289 & 38162.44 & 7.57 & $2.32(1.75-3.06)^{* *}$ & $2.37(1.80-3.13)^{* * *}$ \\
\hline
\end{tabular}

Adjusted hazard ratio (HR) $¥$ : multiple analysis of age, area, income level, and comorbidities; PY = person-years; Rate ${ }^{\#}=$ incidence rate in per 1,000 person-years; IRR $=$ incidence rate ratio; ${ }^{*} p<0.05, * * p<0.01, * * * p<0.001$ 
Table 4. Adjusted HRs measured using the multivariate Cox proportional model for patients with migraine associated with IDA and iron supplementation and stratified by age

\begin{tabular}{|c|c|c|c|c|c|c|}
\hline$\leqq 50$ & & & & & & \\
\hline IDA & $\begin{array}{c}\text { Iron } \\
\text { supplementation }\end{array}$ & Event & PY & Rate $^{\#}$ & IRR $(95 \% \mathrm{CI})$ & Adjusted HR $(95 \% \mathrm{CI}) \ddagger$ \\
\hline- & + & 42 & 12675.34 & 3.31 & 1 (reference) & 1 (reference) \\
\hline- & - & 1045 & 206900.72 & 5.05 & $1.52(1.12-2.08)^{* *}$ & $1.63(1.20-2.22) * *$ \\
\hline+ & + & 588 & 79164.72 & 7.43 & $2.24(1.64-3.07)^{* * *}$ & $2.24(1.64-3.07)^{* * *}$ \\
\hline+ & - & 243 & 28757.30 & 8.45 & $2.55(1.84-3.54) * * *$ & $2.93(2.11-4.07)^{* * *}$ \\
\hline \multicolumn{7}{|l|}{$>50$} \\
\hline IDA & $\begin{array}{c}\text { Iron } \\
\text { supplementation }\end{array}$ & Event & PY & Rate $^{\#}$ & IRR $(95 \% \mathrm{CI})$ & Adjusted HR $(95 \% \mathrm{CI}) \ddagger$ \\
\hline- & + & 24 & 9243.95 & 2.60 & 1 (reference) & 1 (reference) \\
\hline- & - & 379 & 144421.21 & 2.62 & $1.01(0.67-1.53)$ & $0.96(0.63-1.45)$ \\
\hline+ & + & 142 & 45752.73 & 3.10 & $1.20(0.78-1.84)$ & $1.20(0.78-1.85)$ \\
\hline+ & - & 100 & 30698.92 & 3.26 & $1.26(0.80-1.96)$ & $1.29(0.83-2.02)$ \\
\hline
\end{tabular}

Adjusted hazard ratio (HR) $\$$ : multivariable analysis of age, area, income level, and comorbidities; PY = person-years; Rate ${ }^{\#}=$ incidence rate in per 1,000 person-years; IRR = incidence rate ratio; $* p<0.05, * * p<0.01, * * * p<0.001$ 
Supplemental Table 1. The International Classification of Headache Disorders, Second Edition criteria (ICHD-II).

ICHD-II criteria

A. Headache present on 15 days/month fulfilling criteria $C$ and $D$

B. Regular overuse for $>3$ months of 1 drugs that can be taken for acute and/or symptomatic treatment of headache

C. Headache has developed or markedly worsened during medication overuse

D. Headache resolves or reverts to its previous pattern within 2 months after discontinuation of overused medication 


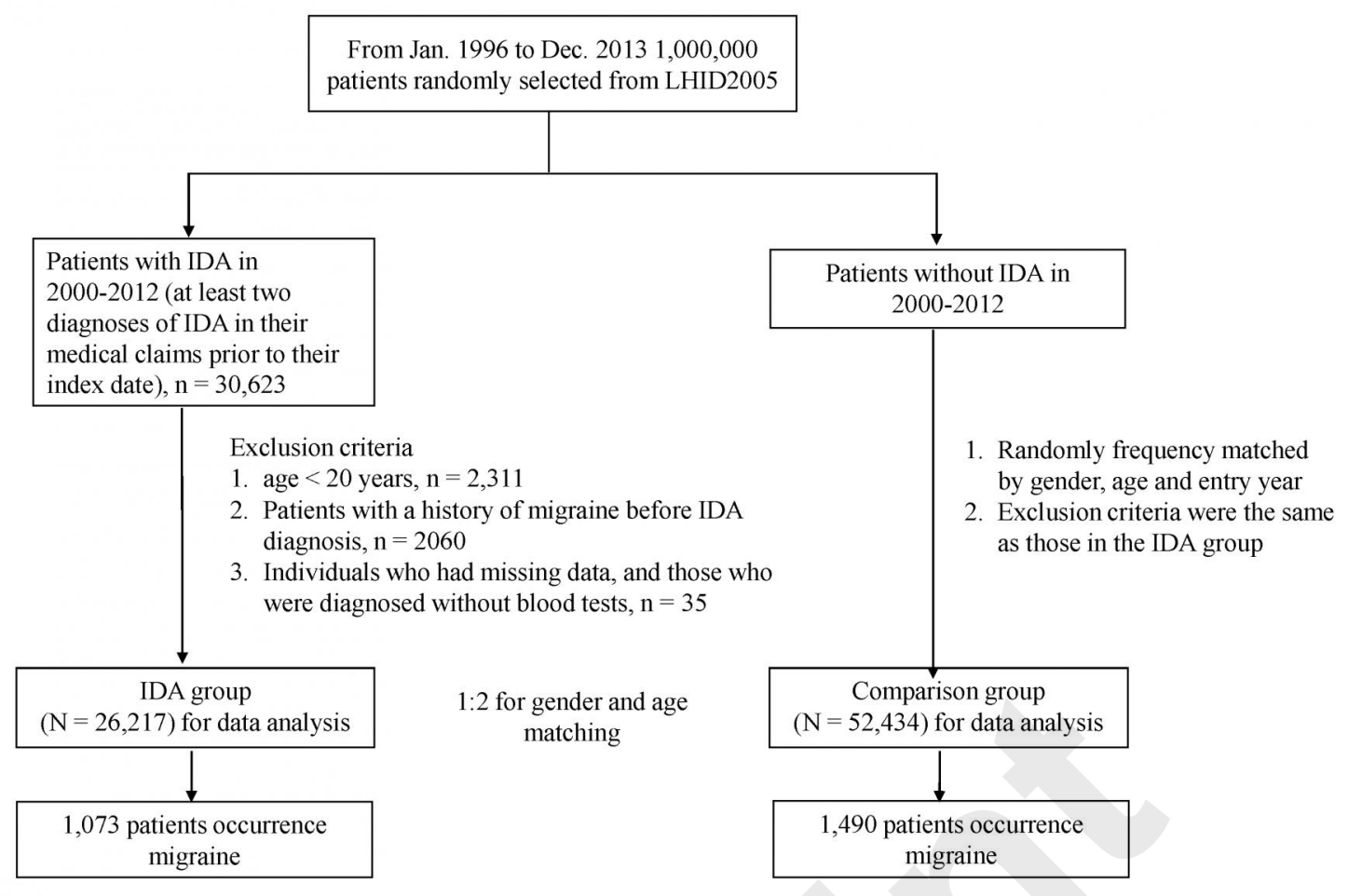

Figure 1. Flow diagram of the present study from the NHIRD. 


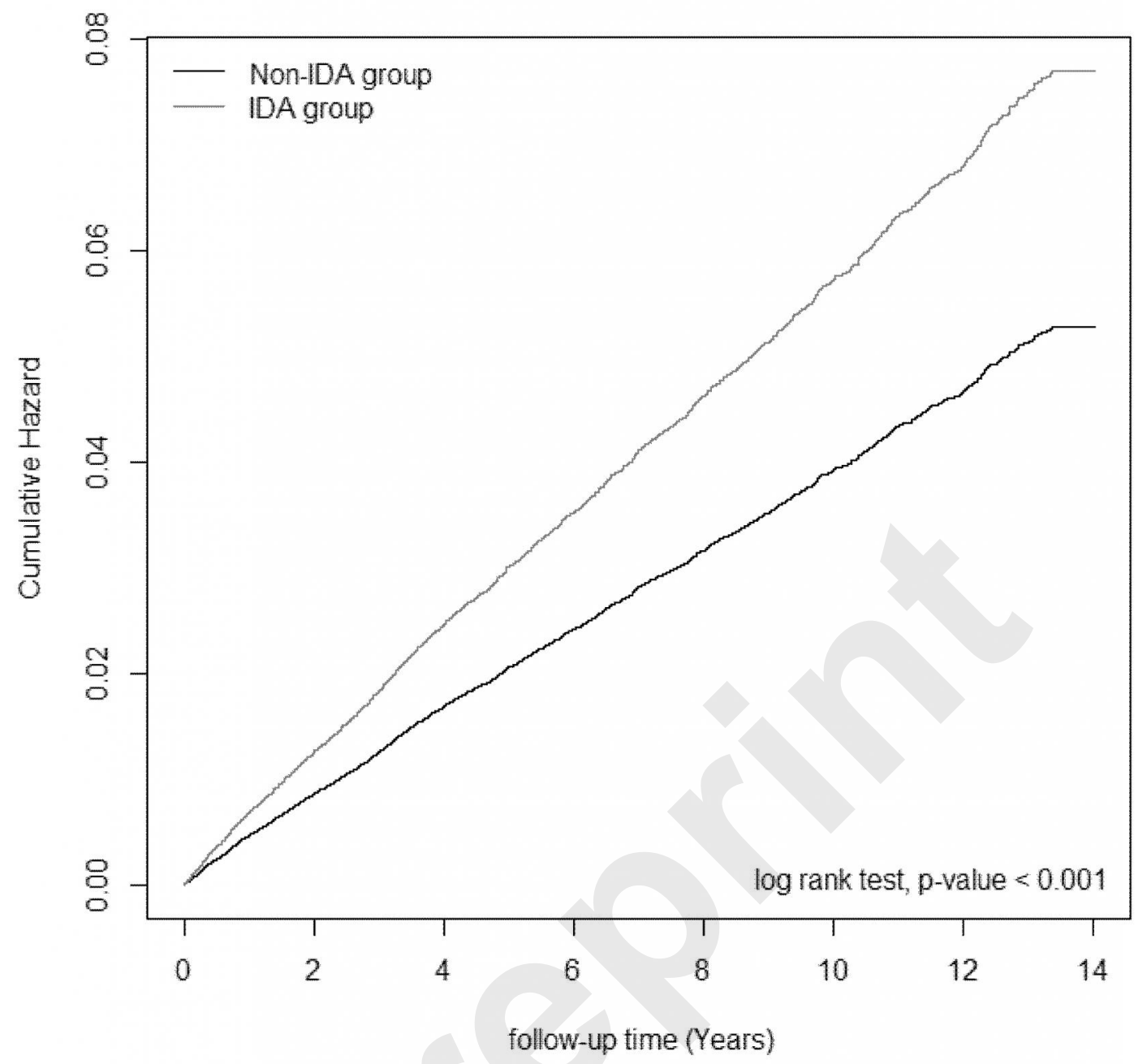

Figure 2. Cumulative incidence of migraine in the IDA and non-IDA groups, compared with a log-rank test. A statistically significant difference exists between the IDA and non-IDA groups $(p<0.001)$ 